\title{
O Uso das Escalas Funcionais para Avaliaçáo Clínica da Distrofia Muscular de Duchenne
}

\author{
The Use of Functional Scales for Clinical Evaluation of Duchenne Muscular Dystrophy \\ Tallita Marins Fittipaldi Barra ${ }^{1}$, Karen Fernanda Baraldi ${ }^{2}$
}

\section{RESUMO}

Distrofia muscular de Duchenne é uma doença genética na qual ocorre uma degeneração progressiva e irreversível do tecido muscular, sendo de rápida evolução. $\mathrm{O}$ tratamento visa preservar o quadro clínico funcional e motor, postergando o processo degenerativo. Objetivo. Demonstrar a aplicabilidade do uso das escalas de Vignos, para o estadiamento da doença, a escala Egen Klassifikation para quantificar o grau de limitaçôes funcionais e respiratórias e a Medida de Função Motora para averiguar a gravidade e progressão da doença. Método. Realizou-se um levantamento bibliográfico de artigos científicos, livros e dissertaçóes publicadas no período de 2000 a 2011, em português, inglês e espanhol. Utilizando as bases de dados Medline, Lilacs e Scielo. Selecionou-se um total de 25 artigos científicos, 1 dissertação e 2 livros. Conclusáo. a aplicabilidade destas escalas auxilia o profissional da saúde a definir as condutas terapêuticas, acompanhar a evolução clínica, avaliar o resultado do tratamento ou a necessidade de alterará-lo, além de possibilitar a realizaçáo de novas pesquisas, com o escopo de fornecer uma melhor qualidade de vida aos portadores da doença.

Unitermos. Distrofia Muscular de Duchenne, Fisioterapia, Avaliação da Deficiência, Transtornos das Habilidades Motoras.

Citaçáo. Barra TMF, Baraldi KF. O Uso das Escalas Funcionais para Avaliação Clínica da Distrofia Muscular de Duchenne.

\begin{abstract}
Duchenne muscular dystrophy is a genetic disease in which there is a progressive and irreversible degeneration of muscle tissue, and rapidly evolving. Treatment aims to preserve the functional and motor status, delaying the degenerative process. Objective. Demonstrate the applicability of using Vignos scales, for the disease stage, Egen Klassifikation, which quantifies the degree of functional and respiratory limitations and Motor Function Measure, which evaluate the seriousness and disease progression. Method. It was conducted a literature review of scientific articles, books and dissertations published from 2000 to 2011, in Portuguese, English and Spanish languages. Using the Medline, Lilacs and Scielo databases, a total of 25 scientific papers, a dissertation and two books was selected. Conclusion. the applicability of the scales mentioned above have the ability to assist health professionals in time to plot and conduct the clinical track. With the continued application of the same it is possible to follow the way the disease progresses during the treatment period and shows an ability to change behavior as needed, and help in carrying out new research, with the aim of providing a higher quality of life.
\end{abstract}

Keywords. Duchenne Muscular Dystrophy, Physiotherapy, Disability Evaluation, Motor Skills Disorders.

Citation. Barra TMF, Baraldi KF. The Use of Functional Scales for Clinical Evaluation of Duchenne Muscular Dystrophy.

\footnotetext{
Trabalho realizado na Universidade Federal de Sáo Paulo - Unifesp, Sáo Paulo-SP, Brasil.

1.Graduada de Fisioterapia, pela Universidade Nove de Julho - Departamento de Ciências da Saúde, São Paulo-SP, Brasil.

2.Fisioterapeuta graduada pela Universidade Metodista de Piracicaba, especialista em Neurologia pela Associação de Assistência à Criança Defeituosa AACD e Mestrado em Neurociências pela Universidade Federal de São Paulo - UNIFESP, São Paulo-SP, Brasil.
}

Endereço para correspondência: Rua Cayowaá, 902, apto. 62 - Perdizes CEP 05018-001, São Paulo-SP, Brasil. E-mail: tallita@gmail.com 


\section{INTRODUÇÃO}

Doenças neuromusculares são conhecidas pelo acometimento primário da unidade motora, podendo também afetar o trato corticoespinal, o cerebelo e as vias espinocerebelares. Estas apresentam comprometimento progressivo em relação à função motora e pulmonar. Dentre as diversas formas de doenças neuromusculares, as mais conhecidas são as distrofias musculares progressivas, que englobam um grupo de doenças genéticas nas quais ocorre degeneração progressiva e irreversível do tecido muscular ${ }^{1,2}$.

A Distrofia Muscular de Duchenne (DMD) é a ocorrência mais comum, com incidência de aproximadamente um em cada 3.500 nascidos vivos masculinos no mundo, sendo transmitida pela mãe. É o tipo mais grave dentre as distrofias musculares, apresentando sinais e sintomas irreversíveis e de rápida evolução ${ }^{2,3}$. Caracteriza-se por um distúrbio genético recessivo, ligado ao cromossomo X, presente na regiāo Xp21. Este codifica a proteína distrofina, essencial para a manutenção da integridade da fibra muscular, garantindo uma contraçáo muscular efetiva ${ }^{4,5}$.

Em média um terço dos pacientes apresenta retardo mental. É possível a visualização precoce - até os 15 meses - do sinal de Gowers, manobra compensatória na qual a criança passa do solo para o ortostatismo "escalando" seu próprio corpo ${ }^{4,6}$. Porém, o mais comum é que sejam evidenciadas as manifestaçôes clínicas por volta dos 3 anos de idade, sendo sua principal característica a fraqueza muscular progressiva, iniciando-se pelos músculos proximais dos membros inferiores, ocasionando dificuldades de locomoção e possíveis quedas, além de complicaçôes para transferências entre localidades ${ }^{2,4}$.

Em torno dos 5 anos é apresentada dificuldade na ascensão de degraus e na corrida. Ocorre ainda um aumento no volume na região do músculo gastrocnêmio, denominado pseudo-hipertrofia. Isto ocorre devido à substituição das fibras musculares por tecido adiposo. Pode ser notada também uma inclinação pélvica e uma acentuaçáo da lordose lombar, adquirindo a marcha anserina ${ }^{4,7}$. Em média, entre os 8 e 12 anos a função da marcha é perdida, o que leva à dependência de cadeiras de rodas para locomoção. Esta situação favorece a obesidade, prejuízo na função dos membros superiores e escoliose - que pode surgir neste momento ou agravar-se quando prévia - gerada pela fraqueza muscular. Esses dois fatores quando juntos acarretam em um comprometimento respiratório e, posteriormente, cardíaco, o que agrava ainda mais o prognóstico da doença $a^{2,4,8}$.

O tratamento da DMD é paliativo. Tem-se empregado, de maneira precoce, o uso de medicamentos e, quando necessário, cirurgias ortopédicas corretivas, buscando lentificar o processo degenerativo ${ }^{5}$. A reabilitação apresenta um papel importante sobre a função motora e pulmonar, que varia de acordo com o estágio clínico do paciente. A fisioterapia, quando inserida na atuação de uma equipe multidisciplinar, apresenta resultados positivos no tratamento da DMD. Esta visa principalmente postergar a evoluçáo dos sintomas, permitindo ao paciente permanecer independente por maior tempo. Objetiva, portanto, promover uma melhora na qualidade de vida do mesmo. Visa também orientar os pais sobre posicionamento articular e postural, no intuito de postergar o aparecimento de deformidades.

As condutas devem ser realizadas de acordo com a faixa etária de cada paciente. Para as crianças é indicada uma atuação lúdica e funcional, o que torna o tratamento menos exaustivo ${ }^{6,9}$. É essencial saber evidenciar o limite de cada paciente, de maneira a não acelerar o processo de fadiga muscular, na qual a degeneração das fibras musculares aconteceria mais rapidamente e atuaria de maneira antagônica ao objetivo proposto ${ }^{10}$.

A avaliação em um paciente com DMD deve analisar de maneira criteriosa todas as alteraçóes decorrentes da doença. Seu beneficio é majorado quando a mesma passa a ser padronizada e validada cientificamente, fornecendo dados numéricos fidedignos importantes para evidenciar a evolução clínica do paciente, bem como os efeitos de alguma terapêutica. Já na avaliação não padronizada cada examinador dará um parecer subjetivo, além de focar no que em sua própria opiniáo possa apresentar maior relevância. Dessa maneira, a confiabilidade dos dados é reduzida para efeito de comparação entre indivíduos ou entre tempos diferentes, o que pode gerar controvérsias no que refere ao tratamento. É primordial que isto seja feito de forma contínua, acompanhando assim a evoluçâo perante o que foi traçado ${ }^{9,11}$.

Em suma, torna-se importante a validade da estrutura da avaliação para permitir a reprodução por pares. 
As escalas utilizam de dados clínicos qualitativos que são convertidos em uma escala numérica. $\mathrm{O}$ uso de escalas funcionais padronizadas e validadas tem o intuito de avaliar a progressão da doença e, para tanto, fornece uma melhor objetividade para efeito de comparação. Dentre elas, está a escala de Vignos ${ }^{12}$, a escala motora Egen Klassifikation $(\mathrm{EK})^{13}$ e a Medida de Função Motora $(\mathrm{MFM})^{14}$. O presente estudo tem como objetivo descrever a aplicabilidade do uso das escalas de Vignos, EK e MFM previamente ao tratamento fisioterápico e global de pacientes com DMD.

\section{MÉTODO}

O estudo consistiu em uma revisão bibliográfica de livros, dissertaçôes e artigos científicos publicados no período de 2000 a 2011, nos idiomas português, inglês e espanhol, realizada nas respectivas bases de dados: Medline, Lilacs e Scielo. Utilizaram-se como critério de busca as seguintes palavras-chaves: Distrofia Muscular de Duchenne, fisioterapia, avaliação da deficiência, distrofina.

\section{RESULTADOS}

Surgiram diversas publicações referentes à DMD, destas, foram selecionados 38 artigos científicos de acordo com uma criteriosa avaliação na qual 13 foram excluídos, devido ao seu foco em alteraçóes ortopédicas e respiratórias, portanto, sem maiores informaçóes essenciais a este trabalho. Desta forma, 25 artigos foram inclusos neste trabalho, destes, 13 em português, 10 em inglês e 2 em espanhol. Em seu conteúdo, 7 referem-se à escala Vignos, 5 à escala EK e 4 à escala MFM, o restante fornece uma visão global sobre a doença.

Na busca por mais dados para este trabalho, 5 dissertaçôes de mestrado foram avaliadas e apenas 1 selecionada para compor, por apresentar em sua pesquisa o uso da escala MFM. Ademais, foram selecionados 2 livros para uso.

\section{DISCUSSÃO}

As Escalas de Vignos, Egen Klassifikation e MFM se destacam, pois permitem avaliaçóes objetivas referentes à função motora - tanto para atividades de locomoção quanto para as de vida diária - ao quadro respiratório instalado e se lida com o indivíduo como um todo, além de serem atualmente as mais citadas na literatura.

\section{Escala de Vignos}

A escala de Vignos foi elaborada por Vignos e Archibald em 1960, modificada em parceria com Spencer em 1963 e modificada pela segunda e última vez por Gardner-Medwin e Walton em $1974^{12}$. É composta por 11 itens a ser pontuados, entre 0 e 10 pontos. Quanto maior a nota obtida, pior é o desempenho funcional. A escala fornece dados de forma classificatória por meio do desempenho de funçóes motoras, graduando por fases o estadiamento da doença ${ }^{15,16}$.

Esta escala é tida como padrão ouro e tornou-se muito utilizada para avaliar a progressão da doença, por tratar-se de um método simples e de fácil aplicação ${ }^{15}$. Porém um estudo, realizado com 15 crianças entre 6 e 10 anos, envolvendo o ato de subir escadas, levantar da cadeira, entre outros, mostrou limitaçóes no resultado da escala, pois a mesma não apresenta detalhes sobre a realização da função, não avaliando as alterações e compensaçôes realizadas durante a execução do movimento ${ }^{17}$.

Para analisar a influência das órteses nos membros inferiores, um estudo feito com pacientes portadores de distrofias musculares, que inclui a DMD, com meninos em média de 12 anos, utilizou a escala de Vignos para avaliar a funcionalidade dos membros inferiores. Demonstrou-se uma aplicação rápida e sem maiores dificuldades. Nesta, 28,5\% dos pacientes foram classificados em grau 9 e 2,8\% entre grau 6 e 8, não foi aplicada em pacientes que não utilizavam órteses. Averiguando a importância da indicação de órteses , pois o seu uso previne deformidades ${ }^{18}$.

Uma pesquisa realizada com 68 pacientes entre 5 e 20 anos, com o intuito de avaliar a correlação da massa da gordura corporal e força muscular, pressōes respiratórias máximas e função motora, utilizou a escala de Vignos e verificou que uma determinada porcentagem de gordura influencia na força muscular, principalmente o sobrepeso, o que afeta a descarga de peso sobre os membros inferiores, e assim prejudica a mobilidade dos mesmos e ocasiona um déficit na realização das funçôes motoras. Por meio dela pode-se associar os fatores citados a fim de proporcionar melhora nas condutas realizadas ${ }^{19}$.

Quanto aos tratamentos farmacológicos, uma pesquisa comparou 19 pacientes em uso de esteróides com outros, de idade parecida, onde o uso não ocorreu. Ob- 
servou-se que o grau funcional, de acordo com a escala de Vignos, se acentuou de maneira significativamente melhor nos pacientes em uso de esteróides que tornou os efeitos colaterais insignificantes relativamente à evolução positiva ocorrida ${ }^{20}$. Em outro estudo associado ao uso de corticóide, 10 crianças, de idade entre 6 e 9 anos - justamente no momento em que a progressão da doença tende levar a um maior acometimento funcional - observou-se uma maior estabilidade no quadro clínico funcional, não houve melhora na realização das funções, mas a duração de estabilidade do quadro se manteve positiva ${ }^{21}$.

Para as terapias por exercícios, houve um estudo de caso baseado nesta escala na qual a mesma não se alterou, realizado apenas com uma criança, no qual o tratamento era composto da aplicação de exercício resistido. $\mathrm{O}$ fato da não alteração indica que esta é uma possível forma de estabilizar o quadro clínico, mantendo-o por maior tempo $^{15}$, mas mais estudos são necessários.

Com isso, vê-se a importância da escala de Vignos, mostrando ao profissional da saúde o estágio apresentado pelo paciente, a fim de uma continuação no tratamento clínico com maior precisão, focado nas alteraçôes presentes. Sendo de aplicação fácil e rápida, não necessita de treinamento para utiliza-la e, ainda, o terapeuta pode conduzi-la durante a sessão. Permite-se também utilizá-la durante a realização de pesquisas em busca de novas formas de lentificar a progressão da doença, e assim, prolongar a independência do paciente.

\section{Escala motora Egen Klassifikation (EK)}

A escala EK foi desenvolvida na Dinamarca e, em outubro de 2006, validada para o português, por Martinez et $\mathrm{al}^{22}$. Visa quantificar o grau de limitação funcional nas atividades de vida diária em pacientes com DMD em fase crônica ${ }^{13,22}$. Possui 10 itens a ser executados. Cada um destes possui 4 alternativas, graduadas de 0 a 3 pontos, a pontuação total pode variar de 0 a 30. Quanto maior o resultado, pior o estado do paciente. O desempenho está relacionado à força muscular periférica, às contraturas musculares, dependência de cadeiras de rodas, e à capacidade vital forçada ${ }^{13}$.

Com a finalidade de averiguar as propriedades da escala validada para o português esta foi utilizada, em 26 pessoas, entre 7 e 22 anos, do sexo masculino, sendo
19 dependentes do uso de cadeira de rodas. Avaliou-se a função de preensão palmar bilateral, envolvendo a força, com o intuito de obter de maneira precisa o grau de acometimento muscular ocasionado pela DMD. Com base nos valores obtidos notou-se a presença de doença muscular periférica. Concluiu-se por meio dos resultados que a empregabilidade da escala é adequada e, além disso, observou-se que apesar da escala ter sido montada para uso em pacientes portadores da cadeira de rodas, sua aplicação também pode ser realizada mesmo quando esta não se faz necessária ${ }^{22}$.

Uma pesquisa envolvendo 9 indivíduos com DMD, sendo 7 portadores de cadeira de rodas e 2 não e, entre eles, 3 dependentes de ventilador mecânico não invasivo, a EK foi escolhida para avaliá-los por ser uma escala de perguntas objetivas e claras, onde se obtém dados importantes sobre a funcionalidade e as atividades de vida diária. Também se mostrou que a mesma pode ser utilizada para pacientes não cadeirantes. Quando comparados os indivíduos nas mesmas funções, houve uma diferença na pontuação da escala, os cadeirantes apresentaram maiores dificuldades na realização das funçóes, outro fator que contribui para essa diferença é a idade, visto que por ser uma doença progressiva, a idade está relacionada com o desempenho funcional, onde quanto maior a idade a tendência é que haja uma maior limitação e, por último, os pacientes que faziam uso de ventilador também apresentaram maior dificuldade nas tarefas ${ }^{23}$.

Além de averiguar a efetividade de determinada abordagem terapêutica, a escala também auxilia a determinar o uso de ventilação mecânica nos pacientes. Desenvolveu-se durante 5 anos uma pesquisa, com avaliaçôes anuais, iniciando-se com 19 participantes, entre 13 e 24 anos, contudo no decorrer do tempo 5 foram a óbito. Antes da pesquisa iniciar, 9 participantes já utilizavam uma modalidade da ventilação mecânica, a pressão positiva contínua nas vias aéreas (CPAP) e 5 passaram a utilizar durante este período. Deste total, para 11 participantes, após 3 anos, foi introduzida a ventilação mecânica assistida via máscara nasal para uso noturno e para 1 deles foi introduzida sem o uso prévio de CPAP, após 12 meses, 4 foram traqueostomizados. A avaliação compreendeu a capacidade funcional e o grau da extremidade superior medida pela EK, a força muscular medida por meio de 
teste manual e a capacidade vital forçada. De todas as variáveis, somente a EK obteve significância estatística, podendo discriminar aqueles que necessitaram de ventilação assistida e os que náo necessitavam, durante todo o período pesquisado ${ }^{24}$.

Outro estudo com a participação de 26 pacientes com DMD utilizou a EK para avaliar a função respiratória, visto que os 3 últimos itens da escala (habilidade para tossir, para falar, bem estar geral) envolvem a questão respiratória. Também se incluiu indivíduos capazes de se locomover sem uso de cadeira de rodas. Com o resultado da pontuação, foi possível prever um alto risco de necessidade de uso de suporte ventilatório não invasivo. Notou-se uma confiabilidade positiva em relação à EK quando se pretende avaliar a função respiratória, isto se deve pela inclusão dos itens sobre a capacidade de tossir, falar e o bem estar ${ }^{25}$.

A escala EK mostrou resultados satisfatórios em todos os estudos que a utilizaram, possui o diferencial de ser a única elaborada pensando na função respiratória e, apesar de feita para usuários de cadeira de rodas, pode-se notar que sua aplicação também se torna favorável em indivíduos que não a utilizam.

\section{Medida de Funçáo Motora (MFM)}

A Escala Medida de função motora foi elaborada por pesquisadores franceses a fim de obter uma nova escala de avaliação para doenças neuromusculares capaz de averiguar a gravidade e progressão da doença e fornecer informaçôes quantitativas em relação às capacidades funcionais de um paciente ${ }^{14}$. Esta escala é adaptada para todos os graus de gravidade, elaborada para pacientes que possuem uma locomoção independente ou não, além de avaliar o paciente como um todo, por meio da movimentação da cabeça, tronco, membros superiores e inferiores $^{16}$.

Inicialmente, foi composta por 51 itens e após algumas mudanças passou a 32 itens, alguns deles estáticos e outros dinâmicos. A avaliação é feita em 3 posiçóes: deitado, sentado ou em pé. Estas são divididas em 3 dimensôes, possibilitando a avaliação das disfunções motoras proximais, distais e axiais. Cada item é graduado entre 0 a 3 pontos. A aplicação dura aproximadamente 36 minutos $^{14}$.
Diferentemente da escala de Vignos e EK, a MFM não é validada especificadamente para a DMD, foi inicialmente validada para distrofias musculares em geral, porém está sendo cada vez mais utilizada pelos profissionais da saúde e pesquisadores em pacientes com DMD. Validou-se para o português em 2006, por Iwabe et al., na qual a versão demonstrou boa confiabilidade, e confirmou seu beneficio em uma avaliação de função motora em doenças neuromusculares. Os 32 itens presentes na escala apresentam objetividade em relação a posicionamentos, movimentos e transferências, sendo de fácil entendimento e aplicação ${ }^{26}$.

Um trabalho realizado com o intuito de acompanhar e prever a perda da deambulação utilizou a MFM para avaliar se a mesma era sensível em identificar alterações na função motora em pacientes com DMD, e capaz de prever perda da deambulação um ano antes do acontecimento, para que o paciente e seus familiares possam se preparar, emocionalmente e financeiramente, para lidar com o momento. Verificou-se neste estudo que foi possível prever a perda da capacidade de deambulação dentro do período de um ano por meio dos dados da Dimensão 1 (que avalia a posição bípede e transferências) quando esta estivesse perto de $40 \%$ ou a pontuação total perto de $70 \%{ }^{27}$.

Em um estudo associado ao uso de corticoide, feito com 33 pacientes com idades entre 6 e 16 anos, deambuladores e cadeirantes verificou-se a aplicabilidade da escala e a evolução da função motora em pacientes com DMD sob tratamento e para analisar separadamente as funçôes motoras específicas, sendo realizadas com o paciente em supino, sedestação, ortostatismo com e sem apoio. A escala demostrou o diferencial de fornecer dados sobre perdas ou ganhos funcionais, e também mostrar alterações funcionais prejudiciais, indicando assim melhores condutas a ser executadas durante o processo evolutivo da doença, no intuito de conseguir prolongar a permanência das funçóes motoras ${ }^{28}$.

Realizou-se um estudo, no período de 6 meses, com intenção de averiguar se os efeitos de uma baixa intensidade de treinamento físico são benéficos, promovendo preservação da resistência muscular e da capacidade funcional. Ao mostrar os resultados do treinamento físico nos indivíduos com DMD, realizados com resistência do 
próprio corpo, de forma lúdica, buscou-se aumentar a atual visão sobre qual o tipo de treinamento físico deve ser recomendado para obter um resultado eficaz. Utilizou como modo de avaliação a MFM, que se demonstrou sensível para detectar mudanças na função motora no período pesquisado, exibindo, portanto, os ganhos promovidos neste caso $^{29}$.

\section{CONCLUSÃO}

A aplicabilidade das escalas de Vignos, EK e MFM têm capacidade de auxiliar o profissional da saúde no momento de traçar condutas e acompanhar a evolução clínica. Com a aplicação contínua das mesmas torna-se possível acompanhar a maneira que a doença evolui no decorrer do período de tratamento e mostra uma possibilidade em alterar condutas quando necessário, além de auxiliar na realização de novas pesquisas, com o escopo de fornecer uma maior qualidade de vida. Contudo, é escassa a quantidade de artigos referentes às mesmas, demonstrando ser necessário mais estudos por parte dos profissionais da saúde, que permitam avaliar e acompanhar o paciente ao longo da evolução da doença, correlacionando o uso das escalas na avaliação do quadro clínico funcional e motor nos pacientes com DMD.

\section{REFERÊNCIAS}

1.Darabas KC, Comim CM, Tuon L. Análise da funcionalidade e qualidade de vida em pacientes portadores de doenças neuromusculares. Fisioter Bras 2009;10:241-7.

2.Fonseca JG, Machado MJF, Ferraz CLMS. Distrofia muscular de Duchenne: complicaçóes respiratórias e seu tratamento. Rev Cienc Med 2007;16:109-20. 3.Strehle EM. Long-term management of children with neuromuscular disorders. J Pediatr 2009;85:379-84.

4.Oliveira AR, Corrêa FI, Valim MM, Oliveira CS, Corrêa JCF. Determination of muscle fatigue index for strength training in patients with Duchenne dystrophy. Fisioter Mov 2010;23:351-60.

http://dx.doi.org/10.1590/S0103-51502010000300002

5.Cammarata-Scalisi F, Camacho N, Alvarado J, Lacruz-Rengel MA. Distrofia Muscular de Duchenne: presentación clínica. Rev Chil Pediatr 2008;79:495-5. http://dx.doi.org/10.4067/S0370-41062008000500007

6.Frezza RM, Silva SRNS, Fagundes SL. Atualização do tratamento fisioterapêutico das Distrofias Musculares de Duchenne e de Becker. RBPS 2005; 18:41-49. http://dx.doi.org/10.5020/18061230.2005.p41

7.Pena FF, Rosolém FC, Alpino MAS. Contribuição da Fisioterapia para o bem-estar e a participação de dois alunos com Distrofia Muscular de Duchenne no ensino regular. Rev Bras Ed Esp 2008;14:447-62.

8.Gonçalves M, Dylewski V, Chaves ACX, Silva TM, Fávero FM, Fontes SV, et al. Qualidade de vida: análise comparativa entre crianças com distrofia muscular de Duchenne e seus cuidadores. Rev Neurocienc 2008;16:275-79.

9.Otsuka MA, Boffa CFB, Vieira ABAM. Distrofia Musculares - Fisioterapia Aplicada. Rio de Janeiro: Revinter, 2005, 248p.

10.Gevaerd MS, Domenech SC, Júnior NGB, Higa DF, Silva AEL. Alteraçōes fisiológicas e metabólicas em indivíduo com distrofia muscular de Duchenne durante tratamento fisioterapêutico: um estudo de caso. Fisioter Mov 2010;23:93-103.

http://dx.doi.org/10.1590/S0103-51502010000100009

11.O'Sullivan SB, Schmitz TJ. Fisioterapia - Avaliação e Tratamento. $5^{\text {a }}$ ed. São Paulo: Manole, 2010, 1200p.

12. Walton JN, Gardner-Medwin D. Disorders of voluntary muscle. Edinburgh: WaltonnChurchill Livingstone, 1974, 116p.

13.Steffensen B, Hyde S, Lyager S, Mattsson E. Validity of the EK scale: a functional assessment of non-ambulatory individuals with Duchenne muscular dystrophy or spinal muscular atrophy. Physiother Res Inter 2001;6:119-34. http://dx.doi.org/10.1002/pri.221

14.Bérard C, Payan C, Hodgkinson I, Fermanian J. A motor function measure scale for neuromuscular diseases. Construction and validation study. Neuromus Dis 2005; 15:463-70.

http://dx.doi.org/10.1016/j.nmd.2005.03.004

15.Ramacciotti EC, Nascimento CF. Efeito do exercício resistido na função motora do paciente com Distrofia Muscular de Duchenne. Rev Neurocienc 2010;18:341-34.

16.Fernandes LAY, Caromano FA, Hukuda ME, Escorcio R, Carvalho EV. Elaboração e confiabilidade da escala funcional do subir e do descer escada para Distrofia Muscular de Duchenne. Rev Bras Fisioter 2010;14:518-26.

17.Caromano FA, Niitsuma LY, Vainzol M, Zatz M. Correlaçáo entre o tempo de realizaçấo de diferentes atividades físicas por portadores de distrofia muscular de Duchenne. Rev Ter Ocup 2003;14:133-40.

18.Lue YJ, Lin RF, Chen SS, Lus YM. Measurement of the functional status of patients with different types of muscular dystrophy. Kaohsiung J Med Sci 2009;25:325-32.

http://dx.doi.org/10.1016/S1607-551X(09)70523-6

19. Caromano FA, Gomes ALO, Pinto NA, Góes ER, Hirosue LN, Assis SMB, et al. Correlação entre massa de gordura corporal, força muscular, pressões respiratórias máximas e função na Distrofia Muscular de Duchenne. Fisioter Mov 2010;23:221-27.

http://dx.doi.org/10.1590/S0103-51502010000200006

20.Schara UMD, Mortier MD, Mortier WMD. Long-Term Steroid Therapy in Duchenne Muscular Dystrophy-Positive Results versus Side Effects. J Clin Neuromuscul Dis 2001;2:179-83.

http://dx.doi.org/10.1097/00131402-200106000-00002

21.Rafia S, Pascual-Pascual SI, Martinez-Granero MA, Pascual-Castroviejo I. Efecto de los corticoides en el tratamiento de la distrofia muscular de Duchenne. An Esp Pediatr 2002;57:518-23.

http://dx.doi.org/10.1016/S1695-4033(02)78709-2

22.Martinez JAB, Brunherotti MA, Assis MR, Sobreira CFR. Validação da escala motora funcional EK para a língua portuguesa. Rev Assoc Med Bras 2006;52:347-51.

http://dx.doi.org/10.1590/S0104-42302006000500024 
23. Okama LO, Queiroz PD, Spina LR, Miranda MBL, Curtarelli MB, Júnior MF, et al. Avaliação funcional e postural nas distrofias musculares de Duchenne e Becker. ConScientiae Saúde 2010;9:649-58.

24.Steffensen BF, Lyager S, Werge B, Rahbek J, Mattsson E. Physical capacity in non-ambulatory people with Duchenne muscular dystrophy or spinal muscular atrophy: a longitudinal study. Develop Med Child Neurol 2002;44:62332. http://dx.doi.org/10.1111/j.1469-8749.2002.tb00847.x

25.Brunherotti MA, Sobreira C, Júnior ALR, Assis MR, Filho JT, Martinez JAB. Correlations of Egen Klassifikation and Barthel Index scores with pulmonary function parameters in Duchenne muscular dystrophy. Heart \& Lung 2007;36:132-9.

http://dx.doi.org/10.1016/j.hrtlng.2006.07.006

26.Iwabe C, Miranda-Pfeilsticker BH, Nucci A. Medida da funçấo motora: versão da escala para o português e estudo de confiabilidade. Rev Bras Fisioter 2008;12:417-24.

http://dx.doi.org/10.1590/S1413-35552008000500012

27.Vuillerot C, Girardot F, Payan C, Fermanian J, Iwaz J, Lattre C, et al. Monitoring changes and predicting loss of ambulation in Duchenne muscular dystrophy with the Motor Function Measure. Devel Med Child Neurol 2010;52:60-5

http://dx.doi.org/10.1111/j.1469-8749.2009.03316.x

28.Silva, EC. Análise da função motora de pacientes com distrofia muscular de Duchenne em corticoterapia através da escala MFM (dissertaçáo). São Paulo: Faculdade de Medicina da Universidade de São Paulo; 2010; p.1-115.

29.Jansen M, Groot IJM, Alfen N, Geurts ACH. Physical training in boys with Duchenne Muscular Dystrophy: the protocol of the No Use is Disuse study. BMC Pediatrics 2010;10:55-62. 